This item was submitted to Loughborough's Research Repository by the author.

Items in Figshare are protected by copyright, with all rights reserved, unless otherwise indicated.

\title{
An exploratory investigation into the longevity of pain reduction following multisensory illusions designed to alter body perception
}

\section{PLEASE CITE THE PUBLISHED VERSION}

https://doi.org/10.1016/j.msksp.2019.102080

\section{PUBLISHER}

Elsevier BV

VERSION

AM (Accepted Manuscript)

\section{PUBLISHER STATEMENT}

This paper was accepted for publication in the journal Musculoskeletal Science and Practice and the definitive published version is available at https://doi.org/10.1016/j.msksp.2019.102080

LICENCE

CC BY-NC-ND 4.0

\section{REPOSITORY RECORD}

Preston, Catherine, Helen R Gilpin, and Roger Newport. 2020. "An Exploratory Investigation into the Longevity of Pain Reduction Following Multisensory Illusions Designed to Alter Body Perception”. Loughborough University. https://hdl.handle.net/2134/13292294.v1. 


\section{Highlights}

1. Illusory stretching led to clinically significant analgesia in hand osteoarthritis

2. Illusion-induced analgesia was found to outlast illusion induction

3. Illusory stretching of the arthritic joint increased subjective flexibility

4. A disownership explanation for illusion-induced analgesia was not supported 


\section{Abstract}

Background: Previous research suggests that multisensory body illusions that alter the conscious bodily experience can modulate pain in osteoarthritis, which may be a result of modifying cortical misrepresentations of the painful body part. However, the longevity and underlying mechanisms of such illusion-induced analgesia is unknown.

Objectives: This experimental study aimed to investigate the therapeutic potential of body illusions, specifically examining the longevity of analgesia and effects on subjective joint flexibility. We also aimed to test if illusory-induced analgesia was due to limb disownership, which is also thought to be affected by body illusions.

Method: Multisensory stretch and shrink illusions were used to manipulate mental representations in hand osteoarthritis. Experiment 1 examined longevity of analgesia by comparing pre-illusion pain ratings with post-illusion ratings taken immediately and over a period of four minutes both with and without vision of the manipulated limb. Experiment 2 compared changes in subjective flexibility between the illusion types. Experiment 3 tested whether an illusion that induced a temporary experience of hand loss would indicate limb disownership as a mechanism for modulating pain during body illusions.

Results: Illusion-induced analgesia was found to outlast the direct application of both shrink and stretch illusions. Illusory stretching provided more clinically significant pain reduction along with increased subjective flexibility. Disownership of the limb had no effect on pain ratings.

Conclusions: Illusory stretching of the joints in osteoarthritis may have significant clinical potential in development of future pain treatments. The results are also compatible with theories of cortical involvement of pain in osteoarthritis. 
An exploratory investigation into the longevity of pain reduction following multisensory illusions designed to alter body perception.

Catherine Preston ${ }^{1,2}$, Helen R. Gilpin ${ }^{1,3}$ and Roger Newport ${ }^{1,4}$

1. School of Psychology, University of Nottingham, Nottingham, United Kingdom

2. Department of Psychology, University of York, York, United Kingdom

3. Department of Psychology, Institute of Psychiatry, Psychology, and Neuroscience, King's College London, London, United Kingdom

4. School of Sport, Exercise and Health Sciences, Loughborough University, Loughborough, United Kingdom

Corresponding author:

Dr. Catherine Preston; Department of Psychology, University of York, York, United

Kingdom;Email: catherine.preston@york.ac.uk; Telephone: 01904322910

\section{Conflicts of interest}

Non declared

\section{Ethical Approval}

The study was conducted in accordance with the declaration of Helsinki and approved by

University of Nottingham Ethics Committee

\section{Funding}

This work was supported by a Dunhill Medical Trust Serendipity Award (SA13/0211). 



\title{
An exploratory investigation into the longevity of pain reduction following multisensory illusions designed to alter body perception.
}

\begin{abstract}
Background: Previous research suggests that multisensory body illusions that alter the conscious bodily experience can modulate pain in osteoarthritis, which may be a result of modifying cortical misrepresentations of the painful body part. However, the longevity and underlying mechanisms of such illusion-induced analgesia is unknown. Objectives: This study aimed to investigate the therapeutic potential of body illusions, specifically examining the longevity of pain relief and effects on subjective joint flexibility. We also aimed to test if illusory-induced analgesia was due to limb disownership, which is also thought to be affected by body illusions.
\end{abstract}

Method: Multisensory stretch and shrink illusions were used to manipulate mental representations in hand osteoarthritis. Experiment 1 examined longevity of analgesia by comparing pre-illusion pain ratings with post-illusion ratings taken immediately and over a period of four minutes both with and without vision of the manipulated limb. Experiment 2 compared changes in subjective flexibility between the illusion types. Experiment 3 tested whether an illusion that induced a temporary experience of hand loss would indicate limb disownership as a mechanism for modulating pain during body illusions.

Results: Illusion-induced analgesia was found to outlast the direct application of both shrink and stretch illusions. Illusory stretching provided more clinically significant pain reduction along with increased subjective flexibility. Disownership of the limb had no effect on pain ratings.

Conclusions: Illusory stretching of the joints in osteoarthritis may have significant clinical potential in development of future pain treatments. The results are also 
compatible with theories of cortical involvement of pain in osteoarthritis.

\section{Key Words}

Analgesia; Illusions; Joint Flexibility; Osteoarthritis

\section{Introduction}

Osteoarthritis $(\mathrm{OA})$ is a debilitating condition characterised by cartilage degeneration in the joints. The pain and function loss of OA make it a leading cause of chronic disability in older adults (Guccione et al. 1994). Despite the presence of tissue damage in $\mathrm{OA}$, there is evidence for additional processes contributing to the pain experienced: pain and sensitivity to noxious and non-noxious stimuli do not correlate with the extent of structural damage (Szebenyi et al. 2005; Arendt-Nielsen et al. 2010; Gwilym et al. 2010) and many patients continue to experience pain after complete joint replacement (Beswick et al. 2012).

Adverse side-effects of drug treatments are common, with long-term outcomes variable, undetermined or detrimental (Jordan et al. 2003; Altman 2018; Crofford 2015), making investigations of non-pharmacological therapies important (Hochberg et al. 2012). An exploratory study in 2011 demonstrated substantial analgesia in OA following exposure to multisensory resizing illusions applied to the painful parts of OA hands (Preston and Newport 2011). Illusion-based therapies are found to help alleviate pain in chronic conditions for which that pain is thought to have a cortical component (Boesch et al. 2016; Tsay et al. 2015; Foell et al. 2014). For example, mirror therapy (McCabe et al. 2003) and illusions that reduce the apparent size of the limb (Moseley et al. 2008) can change reported pain in complex regional pain syndrome type 1 (CRPS1) and there is substantial support for the analgesia using 
mirror therapy for phantom limb pain (Ramachandran and Rogers-Ramachandran 1996; Chan et al. 2007; Finn et al., 2017; Whittkopf and Johnson 2017). For OA, illusory resizing of the joint is shown to modulate pain for the hand and knee (Preston and Newport 2011; Themelis and Newport 2018; Stanton et al. 2018). Currently the mechanisms behind such illusion-induced analgesia are unclear, although it is suggested that the pain may be mediated by misrepresentation of the body in the brain that the illusions temporarily correct (Boesch et al. 2016; Tsay et al. 2015; Foell et al. 2014). Indeed, there is evidence that OA patients misperceive the size of their affected hand (Gilpin et al. 2015). However, suitable controls are required to examine the contribution of placebo, context (e.g. experimental environment) (Themelis and Newport 2018), distraction (Malloy and Milling 2010) or sensory processing (e.g. disownership) (McCabe 2011).

A further consideration is longevity of pain relief; it is not known whether illusion-induced analgesia reported in OA was transient because pain ratings were only taken immediately following the illusion, with no post-experiment follow up (Preston and Newport 2011). Stanton et al. (2018) recorded prolonged analgesia for sustained (continuous) illusions and increased analgesia for repeated illusions in the knee, but it is still not clear whether these effects last beyond the immediate application of the illusion. Body illusions found to provide analgesia with other chronic pain conditions, thought to involve cortical reorganisation, find that the effects outlast illusion induction (e.g. Chan et al., 2007). Therefore, the primary purposes of the current study were to replicate the original results and to investigate the longevity of effects. Additionally, a further debilitating symptom of OA is joint stiffness (Hawker et al. 2018). Preston and Newport (2011) reported anecdotal increases of joint flexibility in some participants. Therefore, a secondary aim was to 
investigate whether subjective range of movement (ROM) is modulated by illusions. An alternative explanation for illusion-induced analgesia is disownership of the real limb (McCabe 2011). Illusory ownership over a fake hand is suggested to cause at least partial disownership of the real limb in healthy controls. While such subjective reports of disownership in heathy individuals are generally low (Longo et al. 2008; Preston 2013), physiological changes during illusions that can induce disownership suggest altered sensory processing in the real limb (Barnsley et al. 2011; Moseley et al. 2008) that could potentially also include pain (McCabe 2011). However, recent studies suggest that ownership is retained following illusory limb resizing (Newport et al. 2015) and whether multisensory illusions thought to cause disownership of the real limb modulate pain thresholds remains equivocal (Mohan et al. 2012, Siedlecka et al., 2014). Thus, the effect of a disownership illusion (Newport and Gilpin 2011) on pain ratings was also investigated.

We hypothesized that OA hand pain would be reduced by illusory hand resizing, that the effect would last beyond the immediate application and that subjective ROM of the affected hand would improve. Given the lack of clear evidence, the hypothesis that illusory disownership would change pain was nondirectional.

\section{Materials and Methods}

Participants:

38 participants (11 male, 27 female; range: 49-84 years; mean: 65 years) were recruited from volunteers responding to newspaper advertisements. Those having clinically-diagnosed OA according to clinical criteria used by their health practitioner in the hands, wrists, or fingers for a least 12 months were selected. Exclusion criteria: 
prior knowledge or expectations about the research, history of neurological or psychiatric disorders, operations/procedures that could have damaged peripheral nerve pathways in the limb. All 38 took part in Experiment 1a, 28 in Experiment 1b and 26 in Experiments 2 and 3. Participants gave written informed consent and the study was conducted in accordance with the declaration of Helsinki and approved by University of Nottingham Ethics Committee. Sample size was based on a power analysis conducted using $\mathrm{G}^{*}$ power 3.1 , with a predicted effect size of $d z=.93$ (Preston and Newport 2011), power $=.95$, alpha $=.05$ suggesting a minimum required sample size of 15 participants. Additional participants were recruited to account for attrition.

Materials:

All experiments were conducted using a $60 \mathrm{~Hz}$ MIRAGE multisensory illusion system (Newport et al. 2010; Newport et al. 2009) MIRAGE uses cameras and mirrors, arranged such that the participant views 'live' images of the real hand in the same spatial location as if viewing the hand directly. Images can be manipulated using inhouse software, allowing spatial distortions of the hand to be displayed within $\sim 20 \mathrm{~ms}$.

\section{Design:}

All experiments employed a quasi-experimental one-group pretest-posttest repeatedtreatment design involving a single patient group examining pain (primary outcome variable) and ROM (secondary outcome variable) before and after our experimental manipulation (Harris et al. 2006).

Procedure: 
Experiment 1a

Experiment 1a $(\mathrm{N}=38)$ was designed to replicate previous results (Preston and Newport 2011) whilst manipulating the magnitude of the illusory shrink and stretch. Following 30 seconds' acclimatisation to MIRAGE, each participant received the stretch and shrink illusions (Figure 1) at full (100\% of the original finger length) manipulation and increments $(25 \%, 50 \%, 75 \%$ of the complete manipulation) in different blocks. Two trials for each increment were delivered. The order of blocks was counterbalanced between participants. The order in which the increments were delivered was counterbalanced within participants (ABCDDCBA).
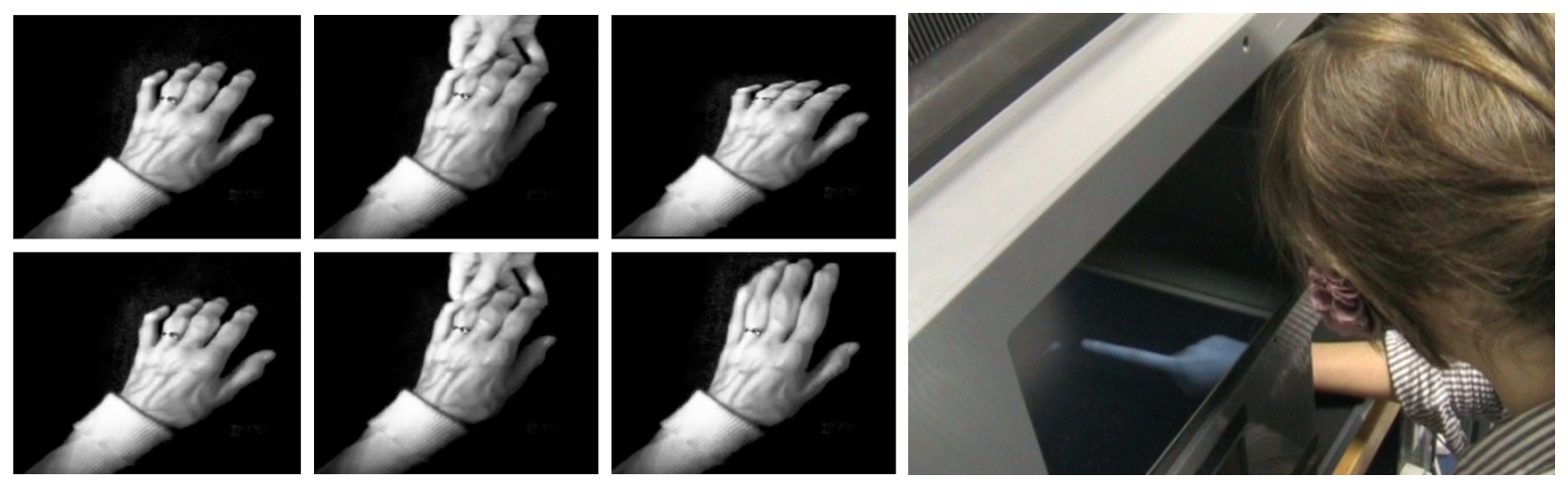

Figure 1: The MIRAGE apparatus depicting a stretch illusion (far right panel); stills from a patient with osteoarthritis undergoing illusory shrinking (top left row) and stretching (bottom left row).

Stretch Illusion: The experimenter gently pulled on the hand/finger distal to the most painful part whilst the image stretched simultaneously. The stretch started at the centre of the painful location and spread longitudinally in both directions. The rate of stretch was controlled by a sliding mechanism, operated by the experimenter, synchronising the timing and speed of the stretch such that the seen stretch and felt pull were perceived at the same time, in addition to increased stretch speed being 
accompanied by a firmer pull.

Shrink Illusion: Shrinking was achieved by gently pushing on the hand/finger, distal to the most painful part whilst the image simultaneously contracted longitudinally. Illusory manipulations (full or incremental) took $\sim 4 \mathrm{~s}$.

To assess the primary outcome measure, verbal pain ratings were taken before and after each full and incremental manipulation using a 21-point numeric rating scale $(\mathrm{NRS})(0=$ no pain at all; $20=$ most severe pain imaginable $)$. A 21 -point rating scale has equivalent reliability to the more frequently used 11-point scale (Jensen and Karoly, 2001) and was chosen in order to make it comparable to the previous study (Preston and Newport 2011). Furthermore, an advantage of using a scale that is likely to differ from a patient's usual routine is that it encourages them to think more about the rating of their current pain (rather than, for example, thinking 'I am always at 10 ').

\section{Experiment 1b:}

Experiment $1 \mathrm{~b}$ examined longevity of pain relief conducted on only those who experienced pain amelioration in Experiment 1a $(\mathrm{N}=28)$. The remaining participants experienced either no change or increased pain. For each participant, the illusion from Experiment 1a that had produced the strongest pain relief was selected: 11 participants underwent shrinking and 17 participants underwent stretching $(2$ of which reported equivalent analgesia for stretching and shrinking but opted for stretching as this was deemed more visually pleasant). After the manipulation, the participant viewed the image of their hand for two minutes, during which time they were 
encouraged to move the hand and fingers. The image was then hidden from view for a further two minutes with participants asked to look towards the hand, though they were unable to see it.

For the primary outcome measure, verbal NRS reports of pain were taken immediately prior to illusion induction, immediately following the manipulation and at 20 -second intervals throughout the subsequent four minutes (two minutes with the hand in view; two hidden). On leaving the laboratory, patients were asked to record the time at which they no longer felt pain-relief and to report this to the experimenter during a telephone follow-up 24 hours later.

\section{Experiment 2}

Experiment 2 examined the effect of illusions on subjective ROM in OA joints. 26 participants from Experiment $1 \mathrm{~b}$ took part; the remainder either unable to attend within the timeframe or declining to participate. Each participant was exposed to the shrink and stretch illusions described above, using the optimal manipulations identified in Experiment 1a. Participants were asked to flex and extend the affected joint for 10 seconds whilst viewing the manipulated hand. Subjective ROM was recorded before the experiment and after each illusion using 21-point NRS $(0=$ cannot move at all; $20=$ complete freedom of movement). Between illusory conditions, participants were asked to remove their hand from MIRAGE and view it directly whilst flexing the joint. The order of the Shrink and Stretch conditions was counterbalanced across participants. In order to retain focus on subjective movement, no pain ratings were made in this experiment. 


\section{Experiment 3}

The contribution of disownership to analgesia in OA was examined using the disappearing hand trick (DHT) (Newport and Gilpin, 2011). Participants held both hands just above the worksurface in MIRAGE. They were to keep the hands still and stop them from touching coloured bars superimposed to either side of each hand that expanded to narrow the available space in which each hand was positioned. During this task ( 25 seconds) the image of each hand moved towards the midline at $25 \mathrm{~mm} / \mathrm{s}$ - a rate too slow to be noticeable. Thus, in order to maintain the appearance of the hands remaining stationary, participants must (unconsciously) move both hands outwards at the same rate, resulting in the hands being located $12.5 \mathrm{~cm}$ further apart than they appear visually. Participants then placed both hands on the worksurface, at which point the superimposed bars and the image of their most painful hand disappeared from view. Participants reached across with their visible hand to try to touch their unseen hand, but due to the hands being further apart than consciously perceived they could only see and feel the empty worksurface, resulting in a powerful sense of hand loss (Newport and Gilpin 2011). Pre- and post-illusion pain ratings were gathered using a 21-point NRS scale assessing the primary outcome measure.

\section{Data Analysis}

As data were ordinal, non-parametric Wilcoxon signed ranks tests and Spearman's rho correlations were used. Effects sizes are reported as r (Pallant 2007). Bonferroni correction was used to correct for multiple comparisons.

Maximum Pain Reduction: To test for illusion-induced analgesia, the lowest reported pain rating following incremental or full manipulations for both illusions in 
experiment 1a was identified for each experiment and compared to pre-manipulation ratings. Change in pain scores were calculated by subtracting the lowest postmanipulation rating from pre-manipulation ratings. Change scores were then compared between illusion types (shrinking and stretching).

Incremental manipulations: To examine the effect of different degrees of the illusory manipulations pre-manipulation pain rating for both illusions were compared to ratings after each increment (averaged across the two trials for each condition) from experiment 1a.

Longevity: To examine how long illusion-induced analgesia lasts pain ratings at each time-point following illusion induction from experiment $1 \mathrm{~b}$ were compared to premanipulation ratings. Pain ratings at the first and final time-points following the manipulation were also compared examining the consistency of analgesia over time.

Subjective Range of Movement (ROM): To test for changes in our secondary outcome variable post-manipulation ROM ratings for each illusion were compared to the pre-illusion rating from experiment 2. To further examine if there is a relationship between analgesia and flexibility, change in ROM scores were calculated by subtracting pre-illusion ratings from post-illusion ratings for both illusion types. Correlations were then conducted between change in ROM scores and change in pain scores calculated from experiment 1a (see above).

Disownership: To examine how an illusion of hand loss influences pain pre-DHT pain ratings were compared to post-DHT ratings from experiment 3. 


\section{Results}

Experiment 1a:

Maximum Pain Reduction: Post-stretch pain ratings (median $=5, \mathrm{IQR}=2-8$ ) were significantly lower than pre-stretch ratings (median $=8, I Q R=5-10)(z=-4.57, p$ $<.001, \mathrm{r}=.52)$, equating to an average reduction of $39.6 \%($ median $=40.8 \%)$.

Furthermore, post-shrink ratings $($ median $=5, \mathrm{IQR}=2.75-10)$ were significantly lower than pre-shrink ratings $($ mean $=6, \mathrm{IQR}=5-11.25)(\mathrm{z}=-4.3, \mathrm{p}>.001, \mathrm{r}=.49)$, equating to an average of $28.1 \%$ pain reduction (median $=15.4 \%)$. No significant difference was found in maximum pain reduction between the illusion types $(\mathrm{z}=$ $2.09, \mathrm{p}=.037, \mathrm{r}=.21) .($ Critical $\mathrm{p}=.017)$. (Figure 2$)$.

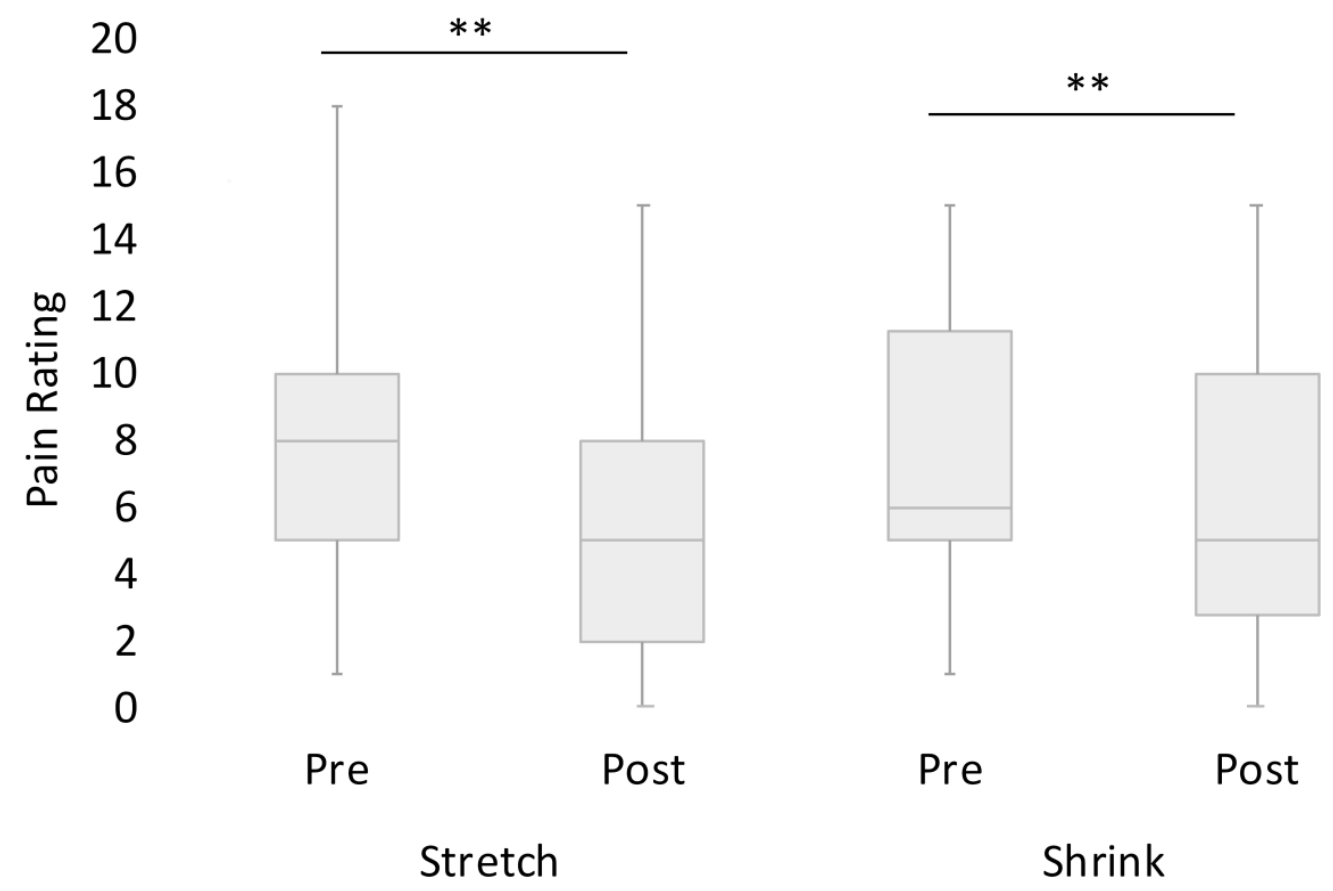

Figure 2: Maximum pain reduction. Box-plots depicting pre- and lowest postillusion pain ratings. Lowest post-manipulation ratings were significantly lower 
than pre-manipulation ratings for both shrink and stretch illusions.

Incremental manipulations: For Stretch, pre-manipulation pain ratings (median $=6.5$, $\mathrm{IQR}=4.9-10.1$ ) were not significantly different from pain reported at $25 \%$ (median $=5.5, \mathrm{IQR}=3-10.1)(\mathrm{z}=-2.404, \mathrm{p}=.016, \mathrm{r}=.28)$, but pain was significantly reduced compared to pre-manipulation scores at 50\% (median $=5, \mathrm{IQR}=2.9-$ $10)(\mathrm{z}=-2.87, \mathrm{p}=.004, \mathrm{r}=3.3)$, and $75 \%($ mean $=5.25, \mathrm{IQR}=2.8-10)(\mathrm{z}=-2.75, \mathrm{p}$ $=.006, \mathrm{r}=3.2$ ). For Shrink, pain ratings were not reduced at any increment. (maximum $\mathrm{z}=-1.895, \mathrm{p}=.058, \mathrm{r}=.22) .($ Critical $\mathrm{p}=.08)$. (Figure 3$)$.
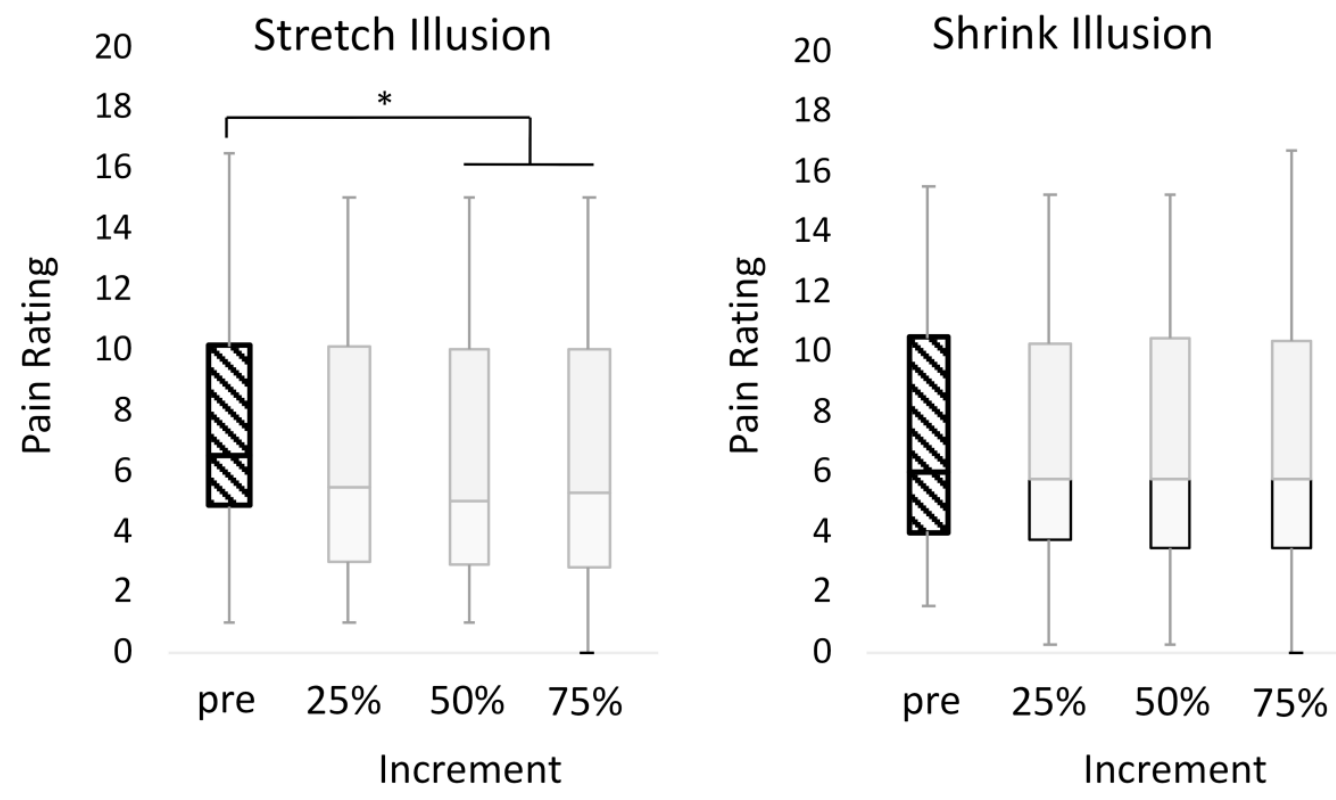

Figure 3: Incremental manipulations: Post-manipulation pain ratings were significantly lower than pre-manipulation at $50 \%$, and $75 \%$ of the maximum illusory Stretch (left panel); there were no significant differences for incremental Shrink illusions (right panel). 
Experiment 1b:

Longevity: Post-manipulation pain ratings were significantly lower than premanipulation at all time-points, including when the hand was hidden from view (smallest $\mathrm{z}=-4.207, \mathrm{p}<.001, \mathrm{r}=.8$ ). The difference in pain ratings between the final $($ median $=5, \mathrm{IQR}=2.25-8)$ and first $($ median $4, \mathrm{IQR}=1.25-7.25)$ postmanipulation time-points was approaching significance $(\mathrm{z}=-3.01, \mathrm{p}=.003, \mathrm{r}=.57)$. (Critical $\mathrm{p}=.003$ ). (Figure 4). Full details of duration of pain relief for each participant can be found in Table S1.

Longevity

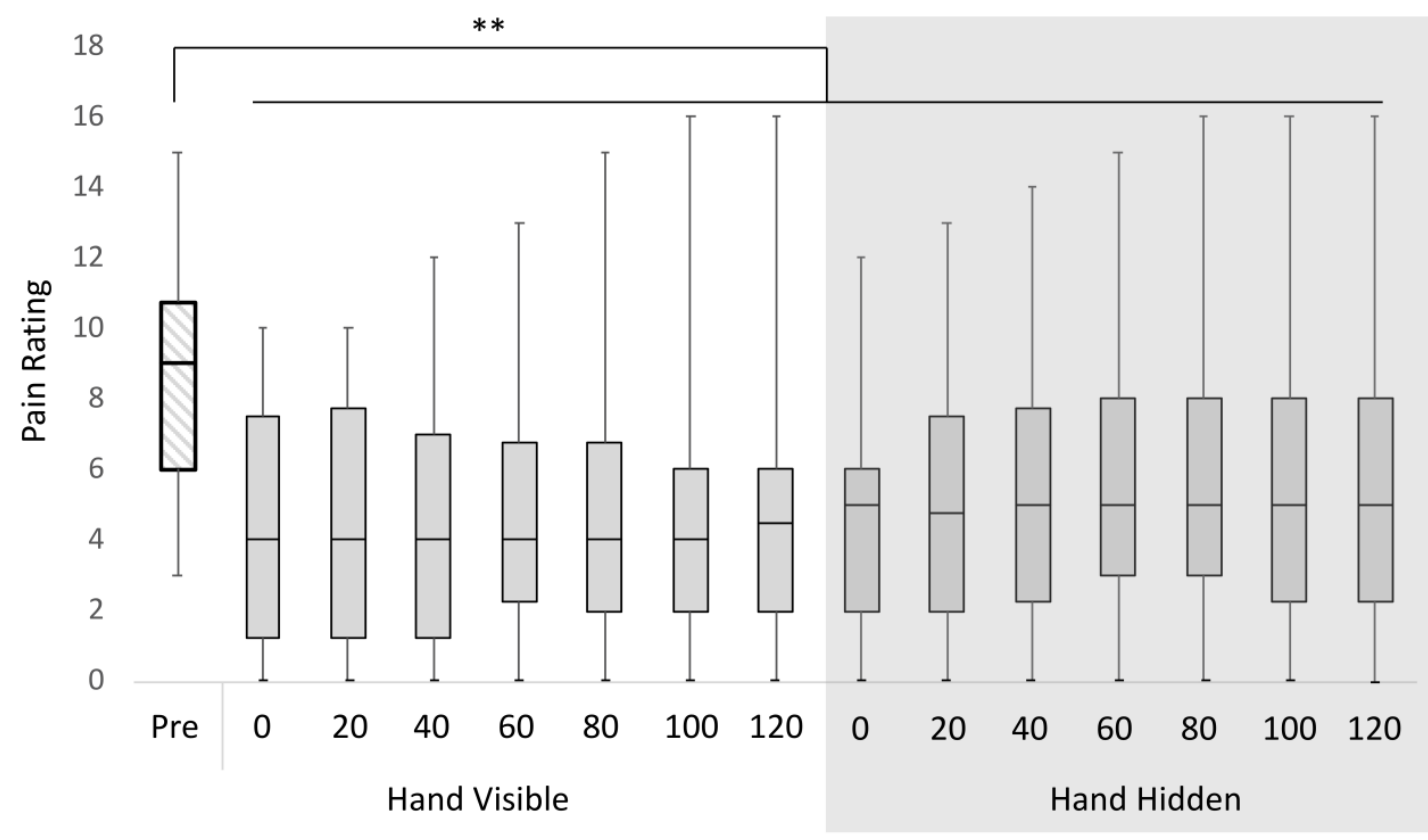

Time in seconds

Figure 4: Longevity of analgesia: pain ratings at pre-manipulation (black bar) were higher compared to each time-point following the illusory manipulation. Pain ratings were taken immediately after the manipulation and at 20 second intevals for two minutes with the hand visible (white background) and for two minutes with the hand occluded (shaded background). There was no significant difference between pain ratings at the first and final time-points following the 


\section{manipulation.}

26 patients were contacted, by telephone, 24 hours after their visit to assess any longer-term changes in pain. Due to a clerical error, follow-up longevity data was not collected for two patients. Three patients provided additional information about the length of pain reduction when they were contacted at a later date for a follow-up event. Pre-manipulation levels of pain returned within 4 minutes for four participants ( $\sim 16 \%)$ and within 20 minutes for 17 participants (68\%). The final $16 \%$ of participants reported pain-relief that outlasted the length of the visit, ranging from 7 hours and 10 weeks (see Table S1).

\section{Experiment 2:}

Subjective range of movement (ROM): ROM ratings were significantly higher after Stretch $($ median $=15, \mathrm{IQR}=12.8-16)$ compared to pre-manipulation $($ median $=13.0$, $\mathrm{IQR}=10-16)(\mathrm{z}=-2.45, \mathrm{p}=.014, \mathrm{r}=.34)$. Ratings following Shrink (median $=12$ $\mathrm{IQR}=10-17)$ were not significantly different to the pre-illusion ratings $(\mathrm{z}=-.438, \mathrm{p}$ $=.662, \mathrm{r}=.06) .($ Critical $\mathrm{p}=.025) .($ Figure 5$)$. Correlations between change in ROM and change in pain for each illusion revealed no significant relationships (stretch: $r_{s}=$ $-.233, \mathrm{p}=.251 ;$ shrink: $\left.r_{s}=.258, \mathrm{p}=.203\right)$. 


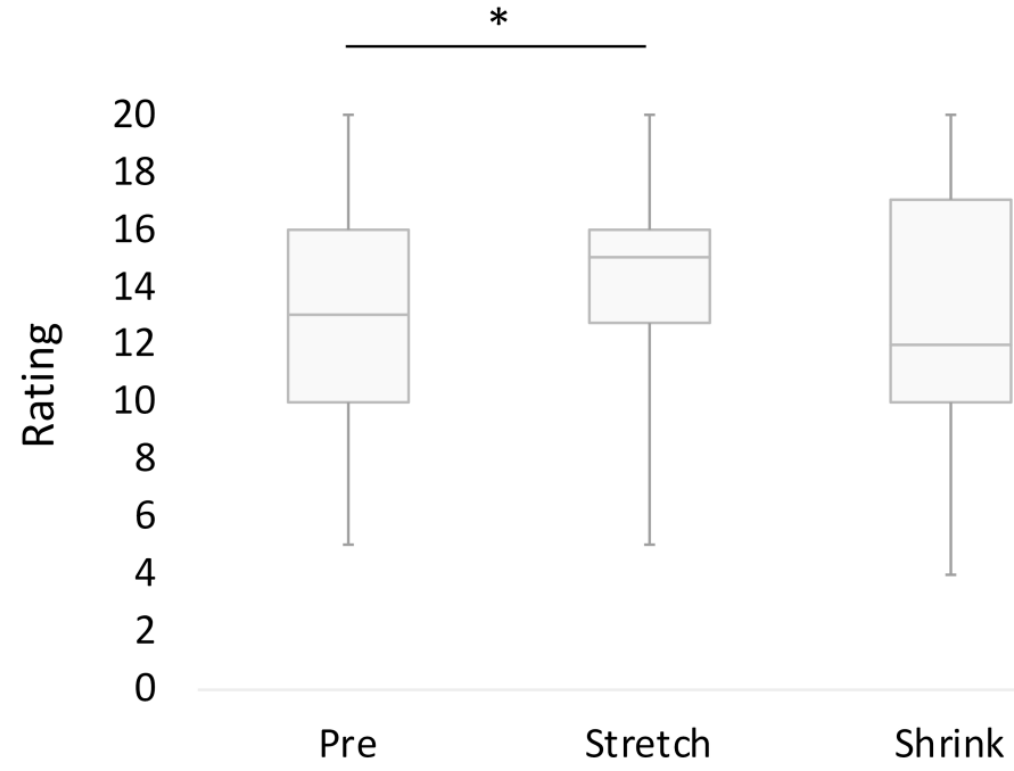

Figure 5: Subjective range of movement (ROM): ROM ratings were significantly greater following Stretch compared to pre-manipulation ratings. There was no significant difference between pre- and post-manipulations ROM ratings for the Shrink illusion.

Experiment 3.

Participants still experiencing complete analgesia from Experiment 2 (pre-DHT pain rating of 0$)$ were excluded from analysis $(\mathrm{N}=2)$. No significant difference between pre- and post-DHT ratings was found $(\mathrm{z}=-.666, \mathrm{p}=.506, \mathrm{r}=.1)$.

\section{Discussion}

These results extend and replicate those of Preston and Newport (2011), demonstrating analgesia following illusory resizing of the painful hand. Importantly, the primary outcome measure of pain relief was not limited to the immediate period of illusion induction, but remained lower for two minutes while viewing the 
manipulated hand, and a further two minutes without vision of the hand. For four participants, pre-manipulation pain returned within four minutes of the experiment; for 17 participants, pain returned within $\sim 20$ minutes; the final four reported some level of analgesia lasting between 7 hours and 10 weeks (see Table S1).

While pain relief was relatively temporary, even brief respite from pain may be beneficial, particularly if associated with increased ROM (observed following illusory Stretch). A temporary window of pain relief could allow patients to engage in physical rehabilitation exercises that might normally be avoided. Further research is required to investigate whether illusions could be used over multiple sessions to tackle long-term pain and disability in OA. A mechanism for this, suggested here and elsewhere (e.g. Preston and Newport 2011; Gilpin et al. 2015; Stanton et al. 2013), is that illusions target a putative cortical body representation component in OA through cortical plasticity. If mental representations of the painful joint are distorted in size (Gilpin et al. 2015), contributing to experienced pain, then multiple sessions of illusory resizing could reduce cortical misrepresentation over time leading to longerterm analgesia. While body illusions can update cortical mapping in healthy controls during illusion induction (Schaefer et al. 2007) a recent finger shrinking study found body representation changes independent of immediate illusion application (Perera et al. 2017), suggesting potential for long-term changes to body representations.

Significant analgesia was observed for both Stretch and Shrink illusions, reducing by $39.6 \%($ median $=40.8 \%)$ and $28.1 \%($ median $=15.4 \%)$ on average respectively. $60 \%$ of participants reported a maximum pain reduction of $>30 \%$ following illusory Stretch and $>40 \%$ of participants reported pain to be reduced by at least $50 \%$. Reductions of $>30 \%$ are considered clinically meaningful and $>50 \%$ extremely meaningful (Dworkin et al. 2018). Although there was no difference in 
maximum analgesia between the illusions, fewer participants reported analgesia at meaningful levels for the Shrink illusion ( $31 \%$ experiencing $>30 \%$ pain reduction; $29 \%$ experiencing $>50 \%$ pain reduction). Furthermore, incremental Shrink was not beneficial and Shrink did not improve perceived flexibility, suggesting that illusory shrinking may be less clinically important compared to illusory stretching. If, as suggested by Gilpin et al. (2015), the representation of the OA hand is too small, illusory stretching could work by correcting shrunken cortical representations. The differences observed for subjective ROM and incremental manipulations between Shrink and Stretch illusions suggest the involvement different underlying mechanisms. It is possible that the effect observed for Shrink may be related attentional factors such as distraction. Anecdotally participants reported that a shrunken finger is unpleasant in appearance compared to a stretched finger. This may explain why smaller increments of Shrink did not elicit a significant effect; disfigurement draws and holds attention, especially within a disease-threat context (Ackerman et al., 2009). The Stretch illusion, however, may tap into (shrunken) body representation mechanisms in OA (Gilpin et al. 2015), or give a perceived relaxation of the joints (especially those with obvious nodes). Illusory stretching has been described as being like 'the finger yawning' or as making the 'whole body relax' (Preston and Newport 2011).

Joint degradation in OA leads to reduced flexibility, which, in turn, increases the progression of joint deterioration, and greater pain and disability (Hawker et al. 2018). Disability is a key contributor to depression (Mossey and Gallagher 2004; Hawker et al. 2018) and reduced quality of life (Salaffi et al. 2005) in OA and thus it is important to help patients keep moving. The current results are the first to demonstrate evidence for increased subjective OA joint flexibility following body illusions. It is unclear 
from the current results whether the observed change in perceived flexibility was a direct effect of the illusion or a secondary consequence of analgesia. Although we did not find correlations between pain and ROM changes, our measures were taken in different experiments on different days. Moreover, if increased ROM was a byproduct of analgesia it may be expected to have a similar effect for the shrinking illusion, which was not observed.

Our results suggest greater clinical potential for stretching relative to shrinking. A visual effect of illusory stretching is the reduced appearance of the joint swelling, a characteristic of OA. It is possible that improving the visual appearance of the hand leads to pain reduction and increased perceived flexibility. Visual appearance of the body is demonstrated to influence sensory processes. In healthy participants, viewing the limb can increase pain thresholds (Longo et al. 2009) and magnification of the viewed arm increases touch sensitivity (Kennett et al. 2001). Additionally, visually minimising a swollen hand can reduce movement induced pain and swelling in CRPS1 (Mossey and Gallagher 2004). Therefore, an alternative account to correcting distorted cortical representations could be that simply making the diseased limb appear visually healthier through reducing the appearance of swelling, may reduce pain.

A previous study has suggested possible mechanisms underlying observed differential effects of shrinking and stretching illusions on tactile perception in healthy individuals (Perera et al. 2015). Specifically, that illusory stretching may temporarily alter cortical processing by increasing activation of multisensory visuoproprioceptive neurons and thus increasing tactile sensitivity. Shrinking, on the other hand, may decrease tactile perception through downregulation of visual information through reduced visual detail from the smaller appearance of the finger. Such 
differing mechanisms may both influence pain in OA, the former through correcting shrunken cortical representations and the latter through down-regulation of sensory information from the finger (Ernst and Banks 2002; Ernst and Bülthoff 2004), including pain, and thus may explain why both produce analgesia but to differing degrees. A further possibility for the different results from shrinking and stretching is via affective networks. Pain is known to involve both perceptual and affective brain regions as part of the pain matrix (Tracey and Mantyh, 2007). Because both Shrink and Stretch illusions involve integration of vision and touch, they may have equivalent effects on sensory perceptual regions resulting in some pain reduction. The enhanced analgesia and flexibility observed following stretching may thus result from additional involvement of affective brain regions, which are more likely to be negatively influenced by shrinking due to the unpleasant appearance of the shrunken finger. Indeed, patients for whom the Shrink and Stretch illusions induced equivalent analgesia maintained a preference for the Stretch illusion as it was deemed more visually pleasant.

Another explanation for illusion-based analgesia in OA is disownership of the real limb (McCabe 2011) through resultant alterations in sensory processing (Barnsley et al. 2011; Moseley et al. 2008). The DHT exploits multisensory integration mechanisms, thought to be essential for the experience of body permanence, to elicit a temporary illusion of limb loss. Here we did not find evidence for analgesia using the DHT. However, while the DHT elicits a feeling of limb disownership in most healthy people, disownership was not measured directly in our OA patients and therefore these results can only be taken as indirect evidence that disownership is unlikely to be the mechanism underlying illusion induced analgesia. 
Although the current results are compatible with a cortical misrepresentation explanation in $\mathrm{OA}$, a limitation of the current research is that, without examining neural activity, the exact processes underlying the observed analgesia are equivocal. Furthermore, although steps were taken to control for participant expectations during recruitment, it is not possible to fully rule out a placebo or contextual effect given the measures and the environment in which the experiments took place. For example, simply sitting in a comfortable room in a distracting (novel) environment for a short time may in itself reduce pain, and whilst this is unlikely to cause the initial sudden pain reduction observed following the illusion induction, it may contribute to the subsequent pain scores and thus exacerbating the apparent longevity of the analgesia. Additionally, although differing results reported here for Shrink and Stretch on ROM and in the previous study for pain (analgesia only when illusions applied to the painful and not non-painful parts of the hand) (Preston and Newport 2011) suggest these effects are not purely contextual, future studies should include specific controls to directly examine this. It should be noted that participants could have interpreted the wording of the ROM statement to mean 'ease' or 'flexibility' of movement rather than magnitude or absolute range. Both are beneficial and future research could measure both constructs explicitly. Finally, although our results are encouraging in terms of clinical impact, these illusions were induced using expensive custom equipment (MIRAGE). Therefore, eliciting these illusions in a clinical or home setting is currently impractical, particularly if pain relief does not exceed 20 mins for most participants. However, recent innovations of such illusions may provide more cost-effective alternatives (Byrne and Preston 2019), which need to be further explored. 
In summary, the current experiments extend and replicate previous findings that demonstrate clinically meaningful analgesia from multisensory illusions in OA by revealing that pain relief lasts beyond the initial manipulation, even when vision of the hand is obscured. Pain reduction was more significant for illusory stretching compared to shrinking and stretching also resulted increased subjective joint flexibility. Taken together these results suggest that continued research to establish whether multisensory illusions can complement existing analgesic treatments for OA is warranted. Furthermore, the results are compatible with growing evidence that distorted cortical representations may contribute to the pain experienced by OA patients, although further neural investigations are essential.

\section{Conflicts of interest}

The authors declare no conflicts of interest

\section{References}

Ackerman, Joshua M, D Vaughn Becker, Chad R Mortensen, Takao Sasaki, Steven L Neuberg, and Douglas T Kenrick. 2009. "A Pox on the Mind: Disjunction of Attention and Memory in the Processing of Physical Disfigurement." Journal of Experimental Social Psychology 45 (3): 478-85. doi:https://doi.org/10.1016/j.jesp.2008.12.008.

Altman, Roy D. 2018. "Intra-Articular Sodium Hyaluronate in Osteoarthritis of the Knee." Seminars in Arthritis and Rheumatism 30 (2). 11-18. doi:10.1053/sarh.2000.0248. Arendt-Nielsen, Lars, Hongling Nie, Mogens B Laursen, Birgitte S Laursen, Pascal Madeleine, Ole H Simonsen, and Thomas Graven-Nielsen. 2010. "Sensitization in 
Patients with Painful Knee Osteoarthritis.” PAIN 149 (3): 573-81.

doi:https://doi.org/10.1016/j.pain.2010.04.003.

Barnsley, N, James H McAuley, R Mohan, A Dey, P Thomas, and G L Moseley. 2011. “The Rubber Hand Illusion Increases Histamine Reactivity in the Real Arm.” Current Biology 21 (23): R945-46. doi:https://doi.org/10.1016/j.cub.2011.10.039.

Beswick, Andrew David, Vikki Wylde, Rachael Gooberman-Hill, Ashley Blom, and Paul Dieppe. 2012. "What Proportion of Patients Report Long-Term Pain after Total Hip or Knee Replacement for Osteoarthritis? A Systematic Review of Prospective Studies in Unselected Patients.” BMJ Open 2 (1): e000435.

Boesch, Eva, Valeria Bellan, G Lorimer Moseley, and Tasha R Stanton. 2016. "The Effect of Bodily Illusions on Clinical Pain: A Systematic Review and Meta-Analysis.” PAIN 157 (3). doi: 10.1097/j.pain.0000000000000423

Byrne, Ailish, and Catherine Preston. 2019. "Mr Fantastic Meets The Invisible Man: An Illusion of Invisible Finger Stretching.” Perception 48 (2). 185-88. doi:10.1177/0301006618821068.

Chan, Brenda L, Richard Witt, Alexandra P Charrow, Amanda Magee, Robin Howard, Paul F Pasquina, Kenneth M Heilman, and Jack W Tsao. 2007. "Mirror Therapy for Phantom Limb Pain.” New England Journal of Medicine 357 (21). 2206-7. doi:10.1056/NEJMc071927.

Crofford, Leslie J. 2015. "Chronic Pain: Where the Body Meets the Brain." Transactions of the American Clinical and Climatological Association 126. American Clinical and Climatological Association: 167-83.

Dworkin, Robert H, Dennis C Turk, Kathleen W Wyrwich, Dorcas Beaton, Charles S Cleeland, John T Farrar, Jennifer A Haythornthwaite, et al. 2018. "Interpreting the Clinical Importance of Treatment Outcomes in Chronic Pain Clinical Trials: IMMPACT 
Recommendations." The Journal of Pain 9 (2). Elsevier: 105-21.

doi:10.1016/j.jpain.2007.09.005.

Ernst Marc O, Heinrich H Bülthoff. 2004. "Merging the senses into a robust percept." Trends Cogn Sci 8 (4). 162-169. doi.org/10.1016/j.tics.2004.02.002

Ernst Marc O, Martin S Banks. 2002. "Humans integrate visual and haptic information in a statistically optimal fashion”. Nature 415 (6870). 429-433. doi.org/10.1038/415429a

Finn Sacha B, Briana N Perry, Jay E Clasing, Lisa S Walters, Sandra L Jarzombek, Sean Curran, Minoo Rouhanian, Mary S Keszler, Lindsay K Hussey-Andersen, Sharon R Weeks, Paul F Pasquina and Jack W Tsao. 2017. “A Randomized, Controlled Trial of Mirror Therapy for Upper Extremity Phantom Limb Pain in Male Amputees.” Front. Neurol. 8: 267. doi.org/10.3389/fneur.2017.00267.

Foell, J, R Bekrater-Bodmann, M Diers, and H Flor. 2014. "Mirror Therapy for Phantom Limb Pain: Brain Changes and the Role of Body Representation.” European Journal of Pain 18 (5): 729-39. doi:10.1002/j.1532-2149.2013.00433.x.

Gilpin, Helen R, G Lorimer Moseley, Tasha R Stanton, and Roger Newport. 2015. “Evidence for Distorted Mental Representation of the Hand in Osteoarthritis." Rheumatology 54 (4): 678-82. http://dx.doi.org/10.1093/rheumatology/keu367.

Guccione, Andrew A, David T Felson, Jennifer J Anderson, John M Anthony, Yuquig Zhang, Peter W Wilson, Margaret Kelly-Hayes, Philip A Wolf, Bernard E Kreger, and William B Kannel. 1994. "The Effects of Specific Medical Conditions on the Functional Limitations of Elders in the Framingham Study." American Journal of Public Health 84 (3): 351-58. doi:10.2105/AJPH.84.3.351.

Gwilym, Stephen. E, Nicola Filippini, Gwenaelle Douaud, Andrew. J Carr, and Irene Tracey. 2010. "Thalamic Atrophy Associated with Painful Osteoarthritis of the Hip Is 
Reversible after Arthroplasty: A Longitudinal Voxel-based Morphometric Study.” Arthritis \& Rheumatism 62 (10): 2930-40. doi:10.1002/art.27585.

Harris Anthony D, Jessina C McGregor, Eli N Perencevich, Jon P Furuno, Jingkun Zhu, Dan E Peterson, Joseph Finkelstein. 2006. "The Use and Interpretation of Quasi-Experimental Studies in Medical Informatics." Journal of the American Medical Informatics Association, 13(1): 16- 23. doi.org/10.1197/jamia.M1749

Hawker, Gillian A, Melissa R French, Ester J Waugh, Monique A M Gignac, C Cheung, and Brian J Murray. 2018. “The Multidimensionality of Sleep Quality and Its Relationship to Fatigue in Older Adults with Painful Osteoarthritis.” Osteoarthritis and Cartilage 18 (11): 1365-71. doi:10.1016/j.joca.2010.08.002.

Hochberg, Marc. C., Roy. D. Altman, Karine Toupin April, Maria Benkhalti, Gordon Guyatt, Jessie McGowan, Tanveer Towheed, Vivian Welch, George Wells, and Peter Tugwell. 2012. "American College of Rheumatology 2012 Recommendations for the Use of Nonpharmacologic and Pharmacologic Therapies in Osteoarthritis of the Hand, Hip, and Knee.” Arthritis Care \& Research 64 (4): 465-74. doi:10.1002/acr.21596.

Jensen Mark P, Karoly, Paul. 2001 “Self-report scales and procedures for assessing pain in adults." In: Handbook of pain assessment, 2nd edition.

Turk, D.C. and Melzack, R. (eds.), The Guilford Press, New York. pp15-34.

Jordan, Joanne M, Gheorghe Luta, Thomas Stabler, Jordan B Renner, Anca D Dragomir, Vladimir Vilim, Marc C Hochberg, Charles G Helmick, and Virginia B Kraus. 2003. "Ethnic and Sex Differences in Serum Levels of Cartilage Oligomeric Matrix Protein: The Johnston County Osteoarthritis Project.” Arthritis \& Rheumatism 48 (3): 675-81. doi:10.1002/art.10822. 
Kennett, Steffan, Marisa Taylor-Clarke, and Patrick Haggard. 2001. "Noninformative Vision Improves the Spatial Resolution of Touch in Humans.” Current Biology 11 (15): 118891. doi:https://doi.org/10.1016/S0960-9822(01)00327-X.

Longo, Matthew R, Viviana Betti, Salvatore M Aglioti, and Patrick Haggard. 2009. "Visually Induced Analgesia: Seeing the Body Reduces Pain.” Journal of Neuroscience 29 (39): 12125-30. doi:10.1523/JNEUROSCI.3072-09.2009.

Longo, Matthew R, Friederike Schüür, Marjolein P M Kammers, Manos Tsakiris, and Patrick Haggard. 2008. "What Is Embodiment? A Psychometric Approach.” Cognition 107 (3): 978-98. doi:10.1016/j.cognition.2007.12.004.

Malloy Kevin M, Leonard S Milling. 2010. “The effectiveness of virtual reality distraction for pain reduction: A systematic review." Clinical Psychology Review 30 (8): 1011-1018. doi.org/10.1016/j.cpr.2010.07.001

McCabe, Candida S, Richard C Haigh, E F J Ring, Peter W Halligan, Patrick D Wall, and David R Blake. 2003. “A Controlled Pilot Study of the Utility of Mirror Visual Feedback in the Treatment of Complex Regional Pain Syndrome (Type 1).” Rheumatology 42 (1): 97-101. http://dx.doi.org/10.1093/rheumatology/keg041.

McCabe, Candida S. 2011. "When Illusion Becomes Reality.” Rheumatology 50 (12): 215152. http://dx.doi.org/10.1093/rheumatology/ker133.

Mohan, Rahul, Karin B Jensen, Valeria I Petkova, Abishikta Dey, Nadia Barnsley, Martin Ingvar, James H McAuley, G Lorimer Moseley, and Henrik H Ehrsson. 2012. "No Pain Relief with the Rubber Hand Illusion.” PLOS ONE 7 (12): e52400. https://doi.org/10.1371/journal.pone.0052400.

Moseley, G Lorimer, Nick Olthof, Annemeike Venema, Sanneke Don, Marijke Wijers, Alberto Gallace, and Charles Spence. 2008. "Psychologically Induced Cooling of a 
Specific Body Part Caused by the Illusory Ownership of an Artificial Counterpart." Proceedings of the National Academy of Sciences 105 (35): 13169-73.

Moseley, G Lorimer, Timothy J Parsons, and Charles Spence. 2008. "Visual Distortion of a Limb Modulates the Pain and Swelling Evoked by Movement." Current Biology 18 (22): R1047-48. doi:https://doi.org/10.1016/j.cub.2008.09.031.

Mossey, Jana M, and Rollin M Gallagher. 2004. "The Longitudinal Occurrence and Impact of Comorbid Chronic Pain and Chronic Depression over Two Years in Continuing Care Retirement Community Residents." Pain Medicine 5 (4): 335-48. http://dx.doi.org/10.1111/j.1526-4637.2004.04041.x.

Newport, Roger., Catherine. Preston, Rachel. Pearce, and Roxanne. Holton. 2009. "Eye Rotation Does Not Contribute to Shifts in Subjective Straight Ahead: Implications for Prism Adaptation and Neglect.” Neuropsychologia 47 (8-9): 2008 - 2012. doi:10.1016/j.neuropsychologia.2009.02.017.

Newport, Roger, Kelly Auty, Mark Carey, Katie Greenfield, Ellen M Howard, Natasha Ratcliffe, Hayley Thair, and Kristy Themelis. 2015. "Give It a Tug and Feel It Grow: Extending Body Perception Through the Universal Nature of Illusory Finger Stretching.” I-Perception 6 (5): 2041669515599310. doi:10.1177/2041669515599310. Newport, Roger, and Helen R Gilpin. 2011. "Multisensory Disintegration and the Disappearing Hand Trick.” Current Biology 21 (19): R804-5. doi:https://doi.org/10.1016/j.cub.2011.08.044.

Newport, Roger, Rachel Pearce, and Catherine Preston. 2010. "Fake Hands in Action: Embodiment and Control of Supernumerary Limbs." Experimental Brain Research 204 (3): 385-95. doi:10.1007/s00221-009-2104-y.

Pallant, Julie. 2007. SPSS Survival Manual : A Step by Step Guide to Data Analysis Using SPSS for Windows. 3rd ed. Maidenhead: Open University Press. 
Perera, A Treshi-marie, Roger Newport, and Kirsten J McKenzie. 2015. "Multisensory Distortions of the Hand Have Differential Effects on Tactile Perception.” Experimental Brain Research 233 (11): 3153-61. doi:10.1007/s00221-015-4384-8.

Perera, A Treshi-marie, Roger Newport, and Kirsten J McKenzie. 2017. "Changing Hands: Persistent Alterations to Body Image Following Brief Exposure to Multisensory Distortions.” Experimental Brain Research 235 (6): 1809-21. doi:10.1007/s00221-0174935-2.

Preston, Catherine. 2013. "The Role of Distance from the Body and Distance from the Real Hand in Ownership and Disownership during the Rubber Hand Illusion." Acta Psychologica 142 (2): 177- 183 doi:10.1016/j.actpsy.2012.12.005.

Preston, Catherine, and Roger Newport. 2011. "Analgesic Effects of Multisensory Illusions in Osteoarthritis.” Rheumatology 50 (12): 2314-15.

http://dx.doi.org/10.1093/rheumatology/ker104.

Ramachandran, Vilayanur S, and Diane Rogers-Ramachandran. 1996. "Synaesthesia in Phantom Limbs Induced with Mirrors." Proceedings of the Royal Society of London. Series B: Biological Sciences 263 (1369): 377 LP-386. doi.org/10.1098/rspb.1996.0058

Salaffi, Fausto, Marina Carotti, Andrea Stancati, and Walter Grassi. 2005. "Health-Related Quality of Life in Older Adults with Symptomatic Hip and Knee Osteoarthritis: A Comparison with Matched Healthy Controls." Aging Clinical and Experimental Research 17 (4): 255-63. http://www.ncbi.nlm.nih.gov/pubmed/16285189.

Schaefer, Michael, Herta Flor, Hans-Jochen Heinze, and Michael Rotte. 2007. "Morphing the Body: Illusory Feeling of an Elongated Arm Affects Somatosensory Homunculus." NeuroImage 36 (3): 700-705. doi:https://doi.org/10.1016/j.neuroimage.2007.03.046. 
Siedlecka Marta, Anna Klimza, Marta Łukowska, Michal Wierzchoń. 2014. "Rubber Hand Illusion Reduces Discomfort Caused by Cold Stimulus." PLoS ONE 9(10): e109909. doi.org/10.1371/journal.pone.0109909

Stanton, Tasha R, Chung-Wei Christine Lin, Helen Bray, Rob J E M Smeets, Deborah Taylor, Roberta Y W Law, and G Lorimer Moseley. 2013. “Tactile Acuity Is Disrupted in Osteoarthritis but Is Unrelated to Disruptions in Motor Imagery Performance.” Rheumatology 52 (8): 1509-19. http://dx.doi.org/10.1093/rheumatology/ket139.

Stanton, Tasha R, Helen R Gilpin, Louisa Edwards, G Lorimer Moseley and Roger Newport 2018. "Illusory resizing of the painful knee is analgesic in symptomatic knee osteoarthritis.” PeerJ, 6, e5206. https://doi.org/10.7717/peerj.5206

Szebenyi, Béla, Anthony. P Hollander, Paul Dieppe, Brian Quilty, John Duddy, Shane Clarke, and Kirwan John. R. 2005. “Associations between Pain, Function, and Radiographic Features in Osteoarthritis of the Knee." Arthritis \& Rheumatism 54 (1): 230-35. doi:10.1002/art.21534.

Themelis Kirsty, and Roger Newport 2018. "An investigation of contextual factors in the application of multisensory illusions for analgesia in hand osteoarthritis." Rheumatology Advances in Practice, 2(2): rky019. https://doi.org/10.1093/rap/rky019

Tracey Irene, Patrick W Mantyh. 2007. “The Cerebral Signature for Pain Perception and Its Modulation.” Neuron. 55 (3): 377-391. doi.org/10.1016/j.neuron.2007.07.012

Tsay, Anthony, Trevor J Allen, Uwe Proske, and Melita J Giummarra. 2015. "Sensing the Body in Chronic Pain: A Review of Psychophysical Studies Implicating Altered Body 
Representation." Neuroscience \& Biobehavioral Reviews 52: 221-32.

doi:https://doi.org/10.1016/j.neubiorev.2015.03.004.

Wittkopf Priscilla G, Mark I Johnson. 2017. "Mirror therapy: A potential intervention for pain management” Rev. Assoc. Med. Bras. 63 (11): 1000-1005.

dx.doi.org/10.1590/1806-9282.63.11.1000 


\begin{tabular}{|c|c|c|c|c|}
\hline Participant & $\begin{array}{l}\text { Pre-pain } \\
\text { returned } \\
\text { within } x \\
\text { minutes }\end{array}$ & $\begin{array}{l}\text { Pain at end of } \\
\text { the session? }\end{array}$ & $\begin{array}{l}\text { Pain at follow } \\
\text { up ( } 24 \text { hours)? }\end{array}$ & $\begin{array}{l}\text { Notes (additional } \\
\text { follow up) }\end{array}$ \\
\hline P2 & 20 & $Y$ & $Y$ & \\
\hline P3 & 20 & $Y$ & $Y$ & \\
\hline $\mathrm{P} 4$ & 20 & $\mathrm{Y}$ & $\mathrm{Y}$ & \\
\hline P5 & 4 & $Y$ & $Y$ & \\
\hline P6 & 4 & $Y$ & $Y$ & \\
\hline P7 & 20 & $Y$ & $Y$ & \\
\hline P9 & 20 & $Y$ & $Y$ & \\
\hline P10 & 20 & $\mathrm{Y}$ & $\mathrm{Y}$ & \\
\hline P12 & 20 & $Y$ & $Y$ & \\
\hline P16 & 20 & $Y$ & $Y$ & \\
\hline P17 & 20 & $Y$ & $Y$ & \\
\hline P18 & 20 & $Y$ & $Y$ & \\
\hline P19 & - & $N$ & $\begin{array}{l}\text { Yes, pain } \\
\text { rating halved }\end{array}$ & $\begin{array}{l}\text { Pain halved for } 3 \\
\text { days }\end{array}$ \\
\hline P20 & - & $\mathrm{N}$ & $N$ & $\begin{array}{l}\text { No pain between } \\
\text { first and second } \\
\text { visit }\end{array}$ \\
\hline P23 & 4 & $Y$ & $Y$ & \\
\hline P24 & 20 & $Y$ & $Y$ & \\
\hline P26 & - & $\mathrm{N}$ & $\mathrm{N}$ & $\begin{array}{l}\text { Reduced pain at } \\
10 \text { weeks, but } \\
\text { stiffness had } \\
\text { returned }\end{array}$ \\
\hline P28 & 4 & $Y$ & $Y$ & \\
\hline P29 & 20 & $Y$ & $Y$ & \\
\hline P31 & 20 & $Y$ & $Y$ & \\
\hline P32 & 20 & $Y$ & $Y$ & \\
\hline P33 & - & $\mathrm{N}$ & Y & $\begin{array}{l}\text { No pain for } 7 \\
\text { hours }\end{array}$ \\
\hline P36 & 20 & $Y$ & $Y$ & \\
\hline P37 & 20 & $Y$ & $Y$ & \\
\hline P38 & 20 & $Y$ & $Y$ & \\
\hline
\end{tabular}

